\title{
Etude de la distribution des composés humiques le long de deux profils sédimentaires continentaux
}

\author{
E. Garnier-Sillam 1 \\ C. Decayeux 1 \\ C. Longuemarel \\ J.M. Bouchyl \\ M. Harry 1
}

Móts clés : Sédiments continentaux, composés humiques, fractionnement de la matière organique.

Le couplage des méthodes de fractionnement granulométrique organo-minéral d'un sédiment et de l'extraction alcaline de ses composés humiques a permis d'étudier la répartition à la fois qualitative et quantitative du carbone dans les différentes fractions de la vase de l'étang des Noës (Yvelines) et de la rivière le Réveillon (Val de Marne).

La comparaison des deux milieux sédimentaires, biologiquement et texturalement dissemblables, a mis en évidence que les processus d'humification et de minéralisation évoluent spatialement de façon différente, comme en témoigne la dégradation différenciée des matières organiques. Les sédiments de l'étang des Noës présentent une évolution de l'humification vers la profondeur, où l'on constate une forte production d'acides humiques, composés fortement polymérisés. En revanche, dans les sédiments du Réveillon, l'humification, prépondérante dès la strate superficielle, subit un blocage dans la strate profonde qui se traduit par une faible production de composés humiques.

La méthodologie employée permet de constater une distribution des substances humiques et de formuler des hypothèses quant à l'évolution des sédiments étudiés.

\section{A study of the distribution of humic compounds along two sedimentary profiles}

Keywords : Continental sediments, humic compounds, fraction of organic matter.

.The coupling of the methods of granulometric analysis of the organo-mineral textural fractions and the alkaline extraction of humic compounds was used to study the distribution, both qualitatively and quantitatively, of carbon in different mud fractions from the pond Noës (Yvelines) and the river Réveillon (Val de Marne).

A comparison of the two sediment environments, biologically and texturally different, showed that the processes of humification and mineralisation evolved spatially in different ways, because of the different breakdown processes of the organic material. The pond sediments from Noës provide a change in humification with depth, where there is a strong production of humic acids that are chiefly polymers. On the other hand, in the sediments of Réveillon, humification is chiefly in the superficial strata and is blocked in the deeper strata with a low production of humic compounds.

The methodology has elucidated the distribution of humic substances and has led to the development of hypotheses for changes in the sediments that were studied.

\section{Introduction}

Malgré la mise en œuvre, ces quinze dernières années, de programmes d'épuration des effluents industriels et domestiques et de plusieurs opérations d'aménagement, l'ensemble du réseau hydrogra-

\footnotetext{
1. Laboratoire de Biologie des Sols et des Eaux, Université Paris XII, 94000 Créteil, France.
}

phique de l'île de France reste très gravement altéré dans toutes ses composantes organiques et inorganiques. L'envasement observé dans un certain nombre de rivières et de plans d'eau atteste du dysfonctionnement de l'écosystème et plus particulièrement du cycle de la matière organique.

Si les composés humiques dans les sols ont été amplement étudiés (Flaig 1970, Harworth 1971, Schnitzer \& Kahn 1978, Andreux 1978, Stevenson 1982, Malcom 1990), il existe peu de travaux consacrés 
aux sédiments lacustres, bien que leur intérêt en matière d'environnement ne soit plus à démontrer (Gjessing 1976, Christman \& Gjessing 1983, Ishiwatari 1985, Fustec et al. 1989). Selon Buffle (1977), il est probable que la réactivité des substances humiques dans le système sédiment-eau interstitielle soit comparable à celle du système sol-eau interstitielle correspondante. Les composés humiques, macromolécules polyfonctionnelles et acides ont un effet biorégulateur dans les sols. Du fait de leur propriétés tensio-actives, ces molécules jouent le rôle d'agents complexants vis-à-vis d'éléments vitaux ou toxiques, minéraux solides (Stevenson 1982, Tan 1974), minéraux dissous (Ong \& Busque 1968, Guy et al. 1975) ou composés organiques (Senesi \& Testini 1982, Choudry 1983). Il en résulte une modification de leur disponibilité dans le milieu par des processus de solubilisation-insolubilisation (Povoledo \& Goltermann 1975, Andreux 1981). Dans un type de sédiment tel que la vase, on peut supposer également que l'accumulation excessive de matières organiques va à l'encontre de la remise en circulation rapide d'éléments nutritifs au profit de la stabilisation et de la condensation de molécules humiques (Faust \& Hunter 1971, Gjessing 1976).

Aussi, la présente étude a-t-elle pour but de caractériser les matières organiques, notamment les matières humiques, présentes dans les sédiments de vase d'un étang, l'étang des Noës (Yvelines) et d'une rivière, le Réveillon (Val de Marne), selon des méthodes d'analyses utilisées en pédologie (Feller 1979). L'intérêt de ce travail repose sur le couplage du fractionnement granulométrique organo-minéral d'un sédiment et de l'extraction alcaline de ses composés humiques. L'étude de la répartition à la fois qualitative et quantitative du carbone dans les différentes fractions humiques extraites doit permettre de caractériser l'état du compartiment organique des différentes strates du sédiment. L'objectif est d'établir un bilan, en un lieu et en un instant donnés, de l'état de la matière organique et de formuler des hypothèses quant à l'évolution de composés humiques soumis à des milieux anthropisés.

\section{Milieux et méthodes}

\subsection{Milieux}

\subsubsection{L'étang des Noës.}

Situation géographique : Cet étang se situe sur la commune du Mesnil Saint Denis (Yvelines), à la limite sud de Saint Quentin en Yvelines et au nord du Parc Naturel de la Haute Vallée de Chevreuse. Cet étang est un des maillons d'un vaste réseau d'étangs et d'aqueducs créé au XVII'e siècle par le maréchal Vauban pour alimenter les jeux d'eau du château de Versailles.

Hydrogéologie : D'une capacité totale de $175000 \mathrm{~m}^{3}$ pour une superficie de 27,5 ha, l'étang reçoit les eaux de 392 ha de bassin versant. Il est alimenté principalement par les eaux provenant de deux rigoles : la rigole de Maurepas et celle dite du « Château ". Son évacuation s'effectue dans la rigole dite « Grand Lit de Rivière » qui rejoint l'étang de Saint-Quentin. L'ensemble de ce site s'étend sur environ 50 ha comprenant le plan d'eau et sa zone périphérique. L'étang des Noës est établi sur des sables granitiques de Lozère très étanches car résultant de la décomposition de feldspaths en argile kaolinique. Cet horizon recouvre les marnes, calcaires et meulières de Beauce. La profondeur moyenne de l'étang est de $87 \mathrm{~cm}$ en dessous du niveau normal 165.40 NGF, la profondeur maximale observée est de $110 \mathrm{~cm}$.

Situation écologique de l'Etang : Celui-ci présente un grand intérêt ornithologique. On recense en effet plus d'une soixantaine d'espèces nicheuses sur les 410 que comprend l'avifaune européenne. Cet étang présente diverses formations végétales qui se sont installées après les travaux de remblaiement résultant du curage de 1974. La qualité écologique de cet étang fait l'objet depuis 1991 d'une étude entreprise par notre laboratoire, depuis la survenue fin octobre 1990 d'une pollution non identifiée qui a occasionné une mortalité piscicole importante. La création très récente d'un dessableur-déshuileur devrait améliorer sensiblement la qualité des eaux reçues lors des pluies, car l'étang des Noës est l'exutoire d'effluents d'eaux pluviales provenant de différents secteurs urbanisés et d'une route à haut trafic (R.N. 10).

Site de prélèvement : Le choix de celui-ci a été dicté par deux impératifs : d'une part, la nécessité d'avoir une certaine accumulation de vase organique afin d'obtenir des résultats significatifs sur un profil différencié, et d'autre part, la nécessité de travailleur sur un échantillonnage repiésentatif de la partie semi-forestière du milieu. De plus, comme aucun curage n'a été entrepris dans cet étang depuis 1974 , on peut considérer que les sédiments déposés n'ont pas été perturbés. Le site de prélèvement 
retenu se situe à 3-4 mètres d'une berge non accessible au public. La campagne de prélèvements a eu lieu en février 1992.

Situation écologique au niveau du site de prélèvement : Sur la berge, une formation de roseau commun, Phragmites communis Trin prédomine sur une largeur d'environ $2 \mathrm{~m}$. On y trouve également des plantes de lieux humides comme Carex pseudocyperus L, Crepis sp., Mentha aquatica L, Poligonum sp. et Solanum dulcamara L. En arrière plan se trouve un massif forestier constitué par un peuplement de reconquête (Betula sp., Populus sp., Salix sp.). Au niveau du site de prélèvement, l'analyse du peuplement en macroinvertébrés montre une faible diversité spécifique. On note la présence d'Odonates, de larves de Diptères Chironomidae (Chironomus sp., Tanytarsini, Tanypodinae), de Trichoptères (Ecnomus sp.), d'Oligochètes Lumbriculidae et Tubificidae, d'Achètes Glossiphonidae et de quelques Gastéropodes Physidae et de Crustacés Asellidae. Il en est de même pour le zooplancton où seuls des cladocères Daphniidae (Daphnia longispina O.F. Mullèr, Daphnia pulex Leydig) et Bosminidae (Bosmina longirostris O.F. Mullèr) et quelques Copépodes (Macrocyclops sp., Tropocyclops sp., Paracyclops sp., Cyclops sp.) sont dénombrés. On peut cependant noter que durant les mois d'avril et mai les Rotifères (Asplancha sp.) deviennent prédominants et que le peuplement estival tend à devenir monospécifique par la prolifération de Daphnia longispina O.F. Mullèr. Le peuplement algal est assez diversifié mais peu abondant. On note la présence de Cyanophycées Nostocales (Anabaena sp.), de Diatomophycées (Surirella sp., Navicula sp.), d'Euchlorophycées Volvocales (Volvox aureus Ehrenberg), de Chlorococcales (Chlorococcum sp., Pediastrum sp. Scenedesmus protuberans Fritsch et Rich), de Zygophycées Zygnématales (Closterium sp.), et d'Ulothricophycées Siphonocladales (Cladophora sp.). Cependant, la dominance dès le mois de mai, d'une algue filamenteuse Spirogyra sp. (Zygophycées, Zygnématales) indique un déséquilibre du milieu. De plus, une analyse microscopique a également révèlé la présence d' $E u$ glena sp.. (Euglenophycées, Eughénales), algue typique des eaux usées et de bactéries, Diffugia sp. et Beggiatoa sp.).

\subsubsection{Le Réveillon}

Situation géographique : Cette rivière, d'une longueur totale de $20 \mathrm{~km}$, prend sa source dans le dépar- tement de la Seine-et-Marne, dans les zones forestières du plateau de Brie, en forêt d'Armanvilliers (en amont de Chevry-Cossigny). Elle traverse ce département sur $6 \mathrm{~km}$ (sur les communes de ChevryCossigny, Férolles-Attily, Lésigny, Ozoir-laFerrière). Ensuite elle traverse le département du Val-de-Marne sur $13 \mathrm{~km}$ (sur les communes de Santenay, Marolles en Brie et Villecresnes) puis celui de l'Essonne (sur la commune de Brunoy et d'Yerres), avant de se jeter dans la rivière l'Yerres.

Hydrogéologie : Le Réveillon repose sur des calcaires marneux de Brie et à meulière, des marnes vertes et supragypseuses et sur du calcaire de Champigny.

Situation écologique du Réveillon : L'envasement formé de matières organo-minérales se présente sous la forme d'une couche fine de quelques centimètres qui colmate le substrat local (graviers et sables). En zone calme, l'épaisseur des sédiments peut atteindre $15 \mathrm{~cm}$. Un envasement important $(>50 \mathrm{~cm}$ ) a été constaté sur le tronçon situé dans la partie en amont du département du Val de Marne, notamment à Santeny, en aval du rejet de la station d'épuration de Sevron (mise en eau en 1969). La qualité de l'eau depuis 1980 est décrite en classe 3 ou hors classe. La pollution du Réveillon est essentiellement due à des rejets d'effluents urbains. En effet, plusieurs stations de Seine-et-Marne rejettent leurs effluents dans le cours d'eau ou dans ses affluents. Les relevés faunistiques et floristiques révèlent une qualité biologique très médiocre et confirment le constat d'une mauvaise qualité générale du cours d'eau.

Site de prélèvement : Celui-ci se situe à $150 \mathrm{~m}$ environ du rejet de la station d'épuration de Sevron dans un secteur recalibré où les eaux sont quasi stagnantes. L'enlisement à ce niveau est important. Les prélèvements ont eu lieu en mars 1992.

Situation écologique au niveau du site de prélèvement : La végétation avoisinante est de type rudéral (jardins et cultures plus ou moins à l'abandon) et de prairie avec une prédominance dans la strate herbacée de l'ortie dioïque (Urtica dioica L.). On trouve entre autres dans cette strate : Anthriscus vulgaris Pers., Rumex aquaticus A.M., Dactylis glomerata L., Poa pratensis L., Bromus sterilis L., Geranium molle L., Cirsium lanceolatum, Scop. Ranunculus sp., Convolvulus arvensis L. et Potentilla reptans $\mathrm{L}$.. La strate arbustive est essentiellement composée de peupliers (Populus sp.), de noyers 
(Juglans regia L.), de saules (Salix sp.), d'aulnes (Alnus glutinosa Gaerta) et de frênes (Fraxinus excelsior L.). L'analyse du biotope révèle une faible diversité biologique au niveau du site de prélèvement. La composition faunistique est réduite à la présence de Trichoptères Hydropsychidae, de larves de Diptères Chironomidae (Chironomus sp., Tanytarsini, Tanypodinae), d'Oligochètes Tubificidae, d'Isopodes Asellidae et de quelques Mollusques bivalves Sphaeriidae. L'indice biotique révèle une qualité médiocre de l'eau (classe 3 dans la grille S.P.E.P.E. de 1971). Le phytoplancton est composé principalement d'Euglenophycées, d'Euglénales (Euglena sp.), d'Euchlorophycées Chlorococcales (Scenedesmus sp.), de Cyanophycées Nostocales (Anabaena sp.) et de quelques Diatomophycées. Des chapelets bactériens de Spaerotilus natans ont été observés, indiquant une surcharge constante en matières organiques.

\subsection{Méthodes}

\subsubsection{Prélèvement des échantillons}

Pour chacun des sites étudiés, trois carottages ont été effectués à l'aide d'un carottier à gravité en PVC de $80 \mathrm{~mm}$ de diamètre, de $1,5 \mathrm{~mm}$ d'épaisseur et de $500 \mathrm{~mm}$ de longueur. Ce préleveur est muni d'une extrémité biseautée possédant une lame métallique coupante et d'une extrémité pourvue d'un système de fermeture étanche. Ces échantillons ont été étudiés frais ou ont été congelés $\left(-18^{\circ} \mathrm{C}\right)$.

Les différentes strates du profil de sédimentation ont été découpées sur des échantillons congelés. Pour chacune des analyses mettant en jeu une strate donnée d'un profil, trois mesures ont été effecutées après homogénéisation d'un aliquote de chacune des trois carottes d'un même site.

\subsubsection{Analyse globale du sédiment}

Profil du sédiment : Les profils sédimentaires des deux milieux étudiés ont fait l'objet d'une description morphologique. Les références des couleurs décrites pour les strates correspondent à celle de l'échelle de Munsell (1990).

Respirométrie : L'intensité des échanges gazeux au sein du sédiment a été étudiée en conditions d'oxygénation au laboratoire. Pour ce faire, après centrifugation d'un aliquote de sédiments frais provenant des strates supérieure et inférieure, $2 \mathrm{~g} \mathrm{sec} \mathrm{de}$ sédiments ont été prélevés puis dilués dans $5 \mathrm{~cm}^{3}$ d'eau.
Pour chaque expérimentation $(\mathrm{n}=3), 2 \mathrm{ml}$ de cette solution ont été placés dans un respiromètre à volume constant (de type Warburg). Le poids sec correspondant aux $2 \mathrm{ml}$ est déterminé. Les paramètres suivants, intensité respiratoire IR (Vol d'O2 consommé/temps/poids de sédiment) et quotient respiratoire $\mathrm{QR}$ (vol de $\mathrm{CO} 2$ dégagé / vol d'O2 consommé) ont été calculés.

Paramètres physico-chimiques: Le $\mathrm{pH}$ et le potentiel d'oxydoréduction (Eh) ont été mesurés in situ dans le sédiment et sur la colonne d'eau le recouvrant. La teneur en eau du sédiment a également été calculée ainsi que la teneur en oxygène dissous de l'eau.

Texture minérale : Afin de connaître la composition du sédiment en particules minérales, une analyse granulométrique en cinq fractions après destruction de la matière organique et emploi de la pipette Robinson a été effectuée d'après la méthode de Duchaufour (1970). Le profil minéral a été établi d'après le diagramme de Jamagne (1967).

Fractionnement granulométrique organominéral : Cette technique décrite par Feller et al. (1983) a été modifiée comme suit : le sédiment séché à l'air libre est homogénéisé à la main et tamisé à $2000 \mu \mathrm{m}$ (fraction $\mathrm{G}^{\prime}$ ). La fraction restante est imbibée d'eau et agitée durant 8 heures avec des billes de verre. Deux tamisages sous eau lui sont ensuite appliqués (à 200 et $50 \mu \mathrm{m}$ ) et conduisent à l'obtention de trois autres fractions, la fraction grossière $\mathrm{G}\left(200\right.$ et $\left.2000_{\mu} \mathrm{m}\right)$, la fraction moyenne M (50 et $200 \mu \mathrm{m})$ et la fraction fine $\mathrm{F}(<50 \mu \mathrm{m})$.

\subsubsection{Analyse de la matière organique}

Dosages du Carbone et de l'Azote : Pour l'ensemble d'une strate et pour chaque fraction granulométrique, les dosages du carbone total selon la méthode d'Anne (1945) modifiée (bichromate de potassium à $2 \%$ en milieu sulfochromique) et de l'azote selon la méthode Kjeldahl, redécrits par Duchaufour (1970), ont été effectués. Le dosage du carbone a été vérifié par un C.H.N.O. (Carlo Erba 1106).

Extractions des composés humiques: Le mode opératoire s'inspire de la méthode proposée par Duchaufour \& Jacquin (1966) modifiée par Bruckert et al. (1974) et Dabin (1976). Celui-ci consiste en extractions successives, en présence de réactifs alcalins, pratiquées sur le sédiment et les différentes 
fractions granulométriques organo-minérales d'une strate donnée. Sur un sédiment broyé et séché, un premier traitement à l'acide phosphorique (2M) est effectué. Celui-ci permet de séparer par densité les débris végétaux dits matières organiques légères (MOL) du reste des constituants et de dissoudre une fraction d'acides fulviques appelés acides fulviques libres ou mobiles (AFLB). Sur le culot lavé à l'eau, un premier traitement à la soude $0,1 \mathrm{~N}$ à froid, à pH12, est effectué et répété jusqu'à épuisement. Après centrifugation $(3500 \mathrm{tr} / \mathrm{mn} ; 30 \mathrm{mn})$, les culots récupérés constituent l'humine (HUM). La séparation des acides fulviques liés (AFLI) et humiques $\mathrm{AH})$ s'effectue par acidification du surnageant à $\mathrm{pH}$ 1,5-1,7 $\left(\mathrm{H}_{2} \mathrm{SO}_{4}, 2 \mathrm{~N}\right)$ qui entraîne la précipitation des $\mathrm{AH}$, les $\mathrm{AF}$ restant en solution. Ces deux fractions sont séparées par centrifugation $(3500 \mathrm{tr} / \mathrm{mn}$; $5 \mathrm{mn}$ ). A l'issue de ces extractions, cing fractions sont obtenues, les matières organiques légères (MOL), les acides fulviques libres (AFLB), les acides fulviques liés (AFLI), les acides humiques (AH), et les humines (HUM) sur lesquelles a été effectué un dosage du carbone total selon la méthode ANNE modifiée (bichromate de potassium à $2 \%$ en milieu sulfochromique).

Les résultats des dosages du carbone seront exprimés :

- en teneur (mg de carbone/g sec de la fraction granulométrique considérée),

- en poids total dans chacune des fractions (mg de carbone/g sec de la fraction granulométrique considérée rapportée à sa granulométrie organominérale)

- et en pourcentage du poids total ou du poids relatif de chaque fraction.

\section{Résultats}

\subsection{Profil vertical du sédiment}

Les descriptions morphologiques des profils sédimentaires des deux milieux étudiés sont présentées sur les figures 1 et 2 .
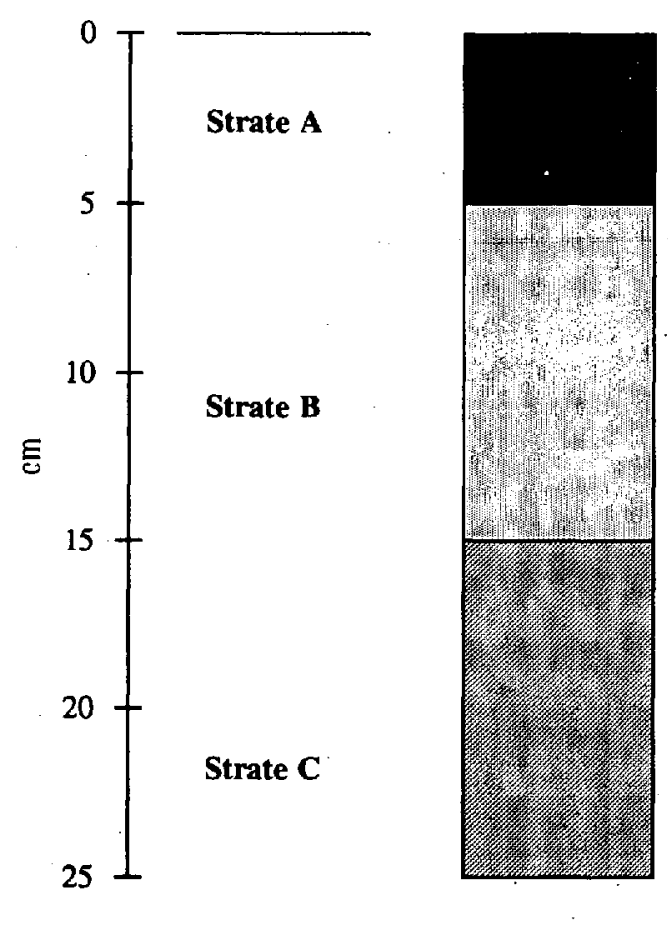

Brun verdâtre (5Y4/1-5Y5/1)

Présence de quelques fragments végétaux de petite taille. reconnaissables à l'oeil nu et d'un léger feutrage racinaire Présence d'argile et de limon, peu de sables

Gris-noir verdâtre (5GY4/1-5GY5/1)

Strate très organique

Présence de nombreux débris végétaux tannés mais non décomposés et d'un feutrage racinaire très noir en surface Présence de graviers, texture limono-argileuse, peu de sables

Ocre-vert (5Y4/1)

Présence de quelques fragments végétaux de grande taille (feuilles, brindilles) et d'une matière organique finement dégradée, clairsemée, non reconnaissable à l'oeil nu Feutrage racinaire léger Présence d'argile en quantité plus importante et de limons Structure polyèdre au séchage.

\section{L'étang des Noës}

Fig. 1. L'Etang des Noës : profil sédimentaire, description morphologique (références des cọuleurs selon celles de l'échelle de Munsell). Fig. 1. L'Etang des Noës : sedimentary profile, morphological description (colour references according to Munsell's scale). 

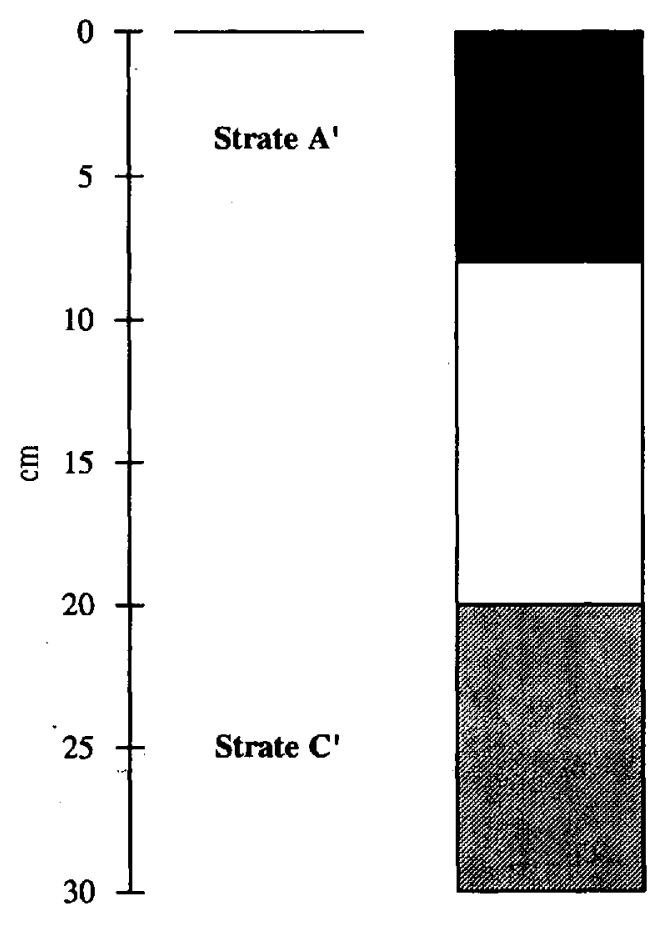

Noir profond uniforme $(7,5 Y R-2 / 0)$

Présence de fragments végétaux de différentes tailles encore reconnaissables à l'oeil nu

Strate très fluide, présence de sables et de limons

Légère odeur d'hydrogène sulfuré

Noir profond uniforme (7,5YR-2/0)

Présence de fragments végétaux tannés mais non dócomposés

Strate dense, pressence de limons, argiles et sables

Forte odeur d'hydrogène sulfuré

\section{Le Réveillon}

Fig. 2. Le Réveillon : (Cf légende Fig. 1).

Fig. 2. Le Réveillon : (for legend see. Fig.1).

Trois strates peuvent être individualisées d'après leur couleur et leur texture, dans le sédiment de l'étang des Noës : la strate supérieure A $(0-5 \mathrm{~cm})$, intermédiaire $B(5-15 \mathrm{~cm})$ et inférieure $C(15-25 \mathrm{~cm})$. Il est à noter que la strate intermédiaire est visiblement la plus riche en matières organiques fraîches et que l'ensemble du sédiment ne dégage auçune odeur particulière.

Le découpage du profil en strates pour le sédiment du Réveillon est moins facile du fait de l'uniformité de couleur noir profond observée sur l'ensemble du profil. En tenant compte des variations de viscosité; de structure et de texture du profil sédimentaire, deux strates ont été retenues, la strate supérieure A' $(0-8 \mathrm{~cm})$ et la strate inférieure $C^{\prime}$ de $(20-30 \mathrm{~cm})$.

\subsection{Analyse globale du sédiment}

\subsubsection{Respirométrie (Tableau 1)}

Etang des Noës : Les intensités respiratoires (IR) sont sensiblement identiques pour les deux strates et les quotients respiratoires (QR) restent environ constants durant le temps de l'expérience. Alors que le quotient respiratoire de la strate supérieure A est en moyenne proche de 1 , il est environ de 0,5 pour la strate inférieure $C$ indiquant un dégagement de $\mathrm{CO} 2$ deux fois plus important que d'O2 consommé.

Le Réveillon : La consommation d'oxygène (IR) est une fois et demie plus importante dans la strate inférieure C' que dans la strate supérieure A'. La production de $\mathrm{CO} 2$ est deux fois plus importante dans la strate $C^{\prime}$ que dans la strate $A^{\prime}$ pendant les 40 premières minutes de l'expérience. Les quotients respiratoires sont pratiquement constants tout le long de l'expérience. Celui-ci est en moyenne deux fois plus élevé dans la strate $A^{\prime}(0,7)$ que dans la strate $C^{\prime}(0,3)$.

\subsubsection{Paramètres physico-chimiques (Tableau 2)}

Les valeurs du potentiel d'oxydo-réduction (Eh) indiquent pour les deux milieux des conditions nettement plus réductrices en profondeur qu'en.surface. 
Tableau 1. Intensité respiratoire (IR, $\mathrm{ml}$ de $\mathrm{O}_{2}$ consommé/g de sédiment sec) et quotient respiratoire ( $\mathrm{QR}, \mathrm{ml} \mathrm{CO}$ dégagé/ml $\mathrm{m}_{2}$ consommé) mesurés en fonction du temps ( $\mathrm{t}$, $\mathrm{mn}$ ) sur les strates des sédiments de l'étang des Noës (strate supérieure $A$, strate inférieure C) et du Réveillon (strate supérieure A', strate inférieure C').

Table 1. Respiratory intensity (IR, $\mathrm{ml}$ of $\mathrm{O}_{2}$ used/g of dry sediment) and respiratory rate (QR, $\mathrm{ml}$ of $\mathrm{CO}_{2}$ released $/ \mathrm{ml}$ of $\mathrm{O}_{2}$ used) measured as a function of time ( $\mathrm{t}, \mathrm{min}$ ) on sediment of the various layers of the sedimentary profile of the "Etang des Noès " (A : upper layer, B : intermediate layer ; C : lower layer) and of « The Réveillon» (A' : upper layer, $C^{\prime}$ : lower layer).

\begin{tabular}{cccccccccc}
\hline \multicolumn{4}{c}{ L'étang des Noës } & \multicolumn{3}{c}{ Le Réveillon } \\
\hline & \multicolumn{3}{c}{ IR } & \multicolumn{2}{c}{ QR } & \multicolumn{3}{c}{ IR } & \multicolumn{2}{c}{ QR } \\
\hline$t(\mathrm{mn})$ & $\mathrm{A}$ & $\mathrm{C}$ & $\mathrm{A}$ & $\mathrm{C}$ & $\mathrm{A}^{\prime}$ & $\mathrm{C}^{\prime}$ & $\mathrm{A}^{\prime}$ & $\mathrm{C}^{\prime}$ \\
\hline 0 & 0 & 0 & 0 & 0 & 0 & 0 & 0 & 0 \\
10 & 42,24 & 72,46 & 0,91 & 0,46 & 73,83 & 76,35 & 0,75 & 0,39 \\
20 & 89,75 & 86,59 & 0,87 & 0,35 & 123,93 & 152,2 & 0,70 & 0,29 \\
30 & 124,75 & 145,11 & 0,97 & 0,46 & 191,73 & 231,45 & 0,54 & 0,29 \\
40 & 155,29 & 178,15 & 0,96 & 0,46 & 206,50 & 262,73 & 0,84 & 0,30 \\
50 & 209,86 & 201,88 & 0,83 & 0,51 & 259,58 & 346,8 & 0,50 & 0,33 \\
\hline 6 & 234,2 & 240,83 & 1,09 & 0,56 & 280,23 & 386,33 & 0,60 & 0,32 \\
\hline 6
\end{tabular}

Tableau 2. Paramètres physico-chimiques et analyse de la texture minérale des sédiments de l'étang des Noës et du Réveillon. Etang des Noës : A, strate supérieure ; B, strate intermédiaire ; $\mathrm{C}$ : strate inférieure. Le Réveillon : $\mathbf{A}^{\prime}$, strate supérieure ; $C^{\prime}$, strate inférieure. Paramètres physico-chimiques : potentiel d'oxydoréduction $(\mathrm{Eh}), \mathrm{pH}$ (mesurés dans les différentes strates des sédiments et sur la colonne d'eau le recouvrant, mesure en surface ; mesure en profondeur), $\mathrm{H}_{2} \mathrm{O} \%$ (teneur en eau du sédiment), $\mathrm{O}_{2} \mathrm{~d}$ (teneur en oxygène dissous de l'eau en mg/ml), DB05 (Demande Biologique Oxygènes à 5 jours en mg/ml) et DCO (Demande Chimique d'oxygène en $\mathrm{mg} / \mathrm{ml}$ ).

Texture minérale des sédiments (après destruction de la matière organique) : $\mathrm{G}^{\prime}$, fraction $>2000 \mu \mathrm{m}$, sables très grossiers ; $\mathrm{G}$, fraction $200-2000 \mu \mathrm{m}$, sables grossiers ; M, fraction $50-200 \mu \mathrm{m}$, sables fins ; F : fraction $10-50 \mu \mathrm{m}$, limons grossiers ; A : fraction $<2 \mu \mathrm{m}$, argiles (en \%).

Table 2. Physico chemical parameters and mineral texture in the layers of the sediments of the « Etang des Noës » and of the « Réveillon ". « Etang des Noës »: A, upper layer ; B, intermediate layer ; C, lower layer. Le Réveillon : A', upper layer ; C' : lower layer. Physico-chemical parameters : oxydo-redox potential, $\mathrm{pH}$ (measured on the various layers of the sediment and on the water column, data in surface ; data in depth), $\mathrm{H}_{2} \mathrm{O} \%$ (water content of the sediment), $\mathrm{O}_{2} \mathrm{~d}$ (dissolved oxygen content in water in mg/ml), $\mathrm{DB} 05$ (biological oxygen demand at 5 days in $\mathrm{mg} / \mathrm{ml}$ ) and DCO (chemical oxygen demand in $\mathrm{mg} / \mathrm{ml}$ ).

Mineral texture of the sediments (after destruction of the organic matter) : G' : fraction $>2000 \mu \mathrm{m}$, very coarse sands ; G, fraction 200-2000 $\mu \mathrm{m}$, coarse sands; $\mathrm{M}$, fraction $50-200 \mu \mathrm{m}$, fine sands ; F : fraction 10-50 $\mu \mathrm{m}$, coarse sitts ; A : fraction $>2 \mu \mathrm{m}$, clays (in $\%$ ).

\begin{tabular}{|c|c|c|c|c|c|c|c|c|c|c|c|c|}
\hline & & Eh (mv) & $\mathrm{pH}$ & $\begin{array}{c}\mathrm{H} 2 \mathrm{O} \\
\%\end{array}$ & $\begin{array}{c}\mathrm{O} 2 \mathrm{~d} \\
\mathrm{mg} / \mathrm{ml}\end{array}$ & $\begin{array}{c}\mathrm{DBO5} \\
\mathrm{mg} / \mathrm{l}\end{array}$ & $\begin{array}{l}\mathrm{DCO} \\
\mathrm{mg} / \mathrm{l}\end{array}$ & $\mathbf{G}^{\prime}$ & $G$ & $\mathbf{M}$ & $\mathbf{F}$ & A \\
\hline & Eau & $253 ; 218$ & 8,$1 ; 7,3$ & & 12,4 & 11,5 & 185 & & & & & \\
\hline Etang des & A & $90 ; 70$ & 6,$9 ; 6,9$ & 58 & & & & 0,5 & 4,6 & 51 & 23,5 & 20,4 \\
\hline \multirow[t]{3}{*}{ Noës } & B & $60 ; 55$ & 6,$8 ; 6,8$ & 49 & & & & 2,8 & 9,7 & 38,2 & 26,1 & 23,2 \\
\hline & $\mathrm{C}$ & 44 & 6,$5 ; 6,3$ & 33 & & & & 1,2 & 3,2 & 36,9 . & 30,7 & 28 \\
\hline & Eau & 155 & $8 ; 1 ; 7 ; 4$ & & $14 ; 1$ & 3 & 72 & & & & & \\
\hline Le & $\mathbf{A}^{\prime}$ & $29 ; 10$ & 7,$5 ; 6 ; 8$ & 79. & & & & 10,0 & 19,4 & $33 ; 5$ & 19,7 & 17,4 \\
\hline Réveillon & B' & $-28 ;-34$ & 6,$4 ; 6 ; 3$ & 56 & & & & 14,3 & $15 ; 3$ & 22,4 & 23,8 & 24,2 \\
\hline
\end{tabular}


Les conditions réductrices (Eh $<+200 \mathrm{mV}$ ) sont atteintes dès la couche supérieure du sédiment $(90$ $\mathrm{mV}$ pour l'étant des Noës, $29 \mathrm{mV}$ pour le Réveillon). Il faut noter que de fortes valeurs négatives sont observées dans la strate profonde du Réveillon $(-28$ à $-34 \mathrm{mV})$.

Comme le potentiel d'oxydo-réduction, les valeurs du $\mathrm{pH}$ montrent une décroissance de la surface de l'eau vers la profondeur du sédiment $(8,11$ à 6,80 pour l'étang des Noës ; 8,10 à 6,30 pour le Réveillon).

Les teneurs en eau interstitielle confirment le phénomène de tassement du sédiment en fonction de la profondeur. Le sédiment du Réveillon apparaît cependant beaucoup plus fluide que celui de l'étang des Noës aussi bien dans la strate supérieure (79\% contre $58 \%$ ) que dans la strate profonde $(56 \%$ contre $33 \%$ ).

\subsubsection{Texture minérale (Tableau 2)}

La texture des trois strates de l'étang des Noës sont de type limono-argileuse avec la présence marquée de sables grossiers dans la strate intermédiaire (B).

Par contre, les deux strates du Réveillon présentent un profil différent : la strate supérieure A' est de type limon-moyen-sableux tandis que la strate profonde $\left(C^{\prime}\right)$ est de type limon-argilo-sableux.

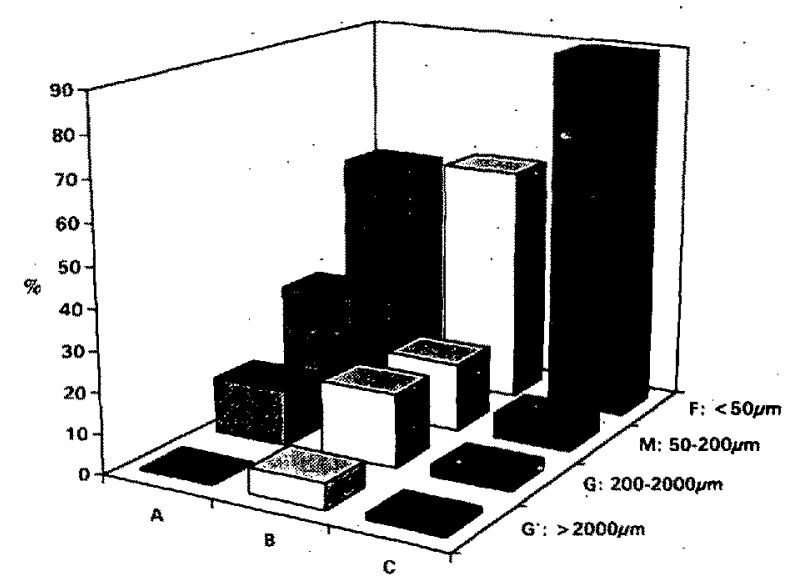

L'Elang des Noes

Fig. 3. Texture organo-minérale des différentes strates du profil sédimentaire de l'Etang des Noës (en pourcentages). A : strate supérieure ; B :-strate.intermédiaire ; C : strate inférieure.

Fig. 3. Organo-mineral texture of the sediment of the various layers of the sedimentary profile of the "Etang des Noës " (in percentages). $\mathrm{A}:$ upper layer ; $\mathrm{B}$ : intermediate layer ; $\mathrm{C}$ : lower layer.

\subsubsection{Fractionnement granulométrique organo-} minéral (Fig. 3 et 4)

Etang des Noës: Les résultats montrent que la fraction $G^{\prime}(<2000 \mu \mathrm{m}$ ) est plus importante dans la strate intermédiaire $B(6,35 \%)$ que dans les strates supérieure $A(2,25 \%)$ et inférieure $C(1,35 \%)$. Ceci est à mettre en relation avec la présence dans cette strate de débris végétaux reconnaissables, peu dégradés, et de quelques graviers. La fraction $\mathrm{G}$ ( 2000 à $200 \mu \mathrm{m}$ ) confirme également cette observation $(12,80 \%$ strate A ; $18,10 \%$ strate B, $2,90 \%$ strate C). En revanche, la fraction moyenne $M$ est présente en plus grande proportion dans la strate supérieure A $(28,45 \%)$ que dans les deux autres strates B $(16,95 \%)$ et $C(6,10 \%)$. La fraction fine $F$, quant à elle, représente la fraction majoritaire dans les trois strates du sédiment et montre une plus grande proportion dans la strate inférieure $C(89,65 \%)$ par rapport aux strates A $(56,50 \%)$ et B $(58,60 \%)$.

Le Réveillon : La texture organo-minérale présente une distribution différente de celle de l'étang des Noës. La fraction G' (> $2000 \mu \mathrm{m}$ ) est présente en proportion plus élevée dans la strate inférieure C' (11 \%) que dans la strate supérieure $A^{\prime}(7 \%)$. De plus, la fraction $G$ est franchement dominante dans la strate $C^{\prime}(46 \%)$ par rapport à la strate $A^{\prime}(25 \%)$.

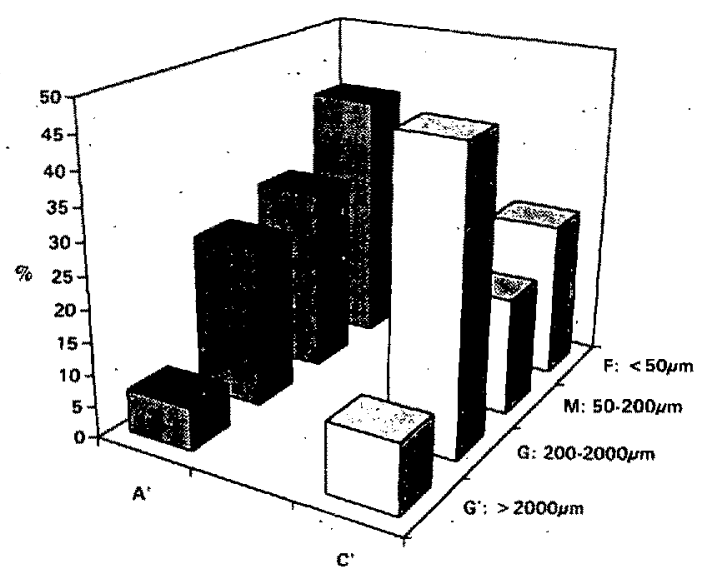

Le Réveilion

Fig. 4. Texture organo-minérale des différentes strates du profil sédimentaire de la rivière, Le Réveillon. A' : stràte supérieure ; $C^{\prime}$ : strate inférieure.

Fig. 4. Organo-mineral texture of the sediment of the various layers of the sedimentary profile of the river « The Réveillon $)$ (in percentages). A': : upper layer, $C^{\prime}:$ lower layer. 
L'examen morphologique du profil sédimentaire a en effet montré la présence persistante de débris végétaux peu dégradés dans la strate inférieure C'. Les fractions $\mathbf{M}$ et $\mathbf{F}$ quant à elles sont en proportion plus importantes dans la strate supérieure $A^{\prime}(28,50 \%$; $39,60 \%$ respectivement) que dans la strate inférieure C' $(18,50 \% ; 24,50 \%$ respectivement).

\subsection{Analyse de la matière organique}

\subsubsection{Dosage du carbone et de l'azote}

L'extraction globale pour le carbone et l'azote se situe à $100 \% \pm 5 \%$, aussi les bilans ont été ajustés à $100 \%$ et répartis proportionnellement dans les différentes strates.

Etang des Noës (Tableau 3) : La teneur en carbone global n'est pas significativement différente entre la strate supérieure $A(24,33 \mathrm{mg} / \mathrm{g})$ et inférieure $B(24,44 \mathrm{mg} / \mathrm{g})$ et diminue d'environ $30 \%$ dans la strate $\mathrm{C}(16,74 \mathrm{mg} / \mathrm{g})$. L'analyse de la teneur en carbone dans les différentes fractions granulométriques organo-minérale des trois strates montre que cette teneur est prépondérante dans la fraction $G$, pour les strates $A(8,74 \mathrm{~m} / \mathrm{g}, 36 \%)$ et $B(8,48 \mathrm{mg} / \mathrm{g}$,
$35 \%$ ) reflétant la quantité de la matière organique fraîche contenue dans des résidus végétaux dont la taille est comprise entre 200 et $2000 \mu \mathrm{m}$. En revanche, la strate $C$ présente une valeur minimale dans cette fraction $\mathrm{G}(1,51 \mathrm{mg} / \mathrm{g}, 9 \%)$. La majeure partie du carbone de cette dernière strate se localise à $63 \%$ dans la fraction fine $\mathrm{F}(10,51 \mathrm{mg} / \mathrm{g})$ alors que les strates $\mathrm{A}$ et $\mathrm{B}$ présentent respectivement $23 \%(5,55 \mathrm{mg} / \mathrm{g})$ et $28 \%(6,84 \mathrm{mg} / \mathrm{g})$ de carbone dans cette fraction F. Il apparaît clairement que l'essentiel du carbone de la strate $C$ est réparti dans les structures de taille beaucoup plus réduites $(<50 \mu \mathrm{m})$ que ne l'est le carbone des strates sus-jacentes.

Le dosage de l'azote global dans les différentes strates montre une faible diminution de la strate supérieure $A(1,41 \mathrm{mg} / \mathrm{g})$ vers la strate inférieure C $(1,30 \mathrm{mg} / \mathrm{g})$. Les dosages de l'azote dans les différentes fractions granulométriques soulignent une évolution globalement identique à celle du carbone. L'étude du rapport $\mathrm{C} / \mathrm{N}$ montre qu'il n'existe pas de grandes différences entre les strates bien que le rapport global diminue avec la profondeur $(17,2$ strate A ; 14,6 strate B ; 12,9 strate $C)$.

Tableau 3. Analyse de la matière organique dans les différentes fractions texturales organo-minérales des strates du sédiment de l'étang des Noës : carbone organique total (COT), azote total (NT), et rapport C/N. a. : teneur (mg de carbone ou d'azote/g sec de la fraction granulométrique considérée), $b$ : poids total dans chacune des fractions ( $\mathrm{x} \%$ texture organo-minérale de la fraction) et $\%$; pourcentage de chaque fraction ; $\mathrm{G}^{\prime}$ : fraction très grossière $>2000 \mu \mathrm{m}$; $\mathrm{G}$ : fraction grossière, $200-2000 \mu \mathrm{m}$; $\mathrm{M}$ : fraction moyenne, $50-200 \mu \mathrm{m} ; \mathrm{F}:$ fraction fine, $<50 \mu \mathrm{m}, \mathrm{Gl}:$ fraction globale.

Table 3. Analysis of the organic matter in the various organo-mineral textural fractions of the sedimentary layers of the « Etang des Noës " : total organic carbon content (COT), total nitrogen content (NT), C/N ratio. a : content (mg of carbon or nitrogen/dry gramme of the granulometric fraction considered), $b$ : total weight in each fraction ( $\mathrm{a} \% \%$ organo-mineral texture) and $\%$; percentage of each fraction ; $\mathrm{G}^{\prime}$ : very rough fraction $>2000 \mu \mathrm{m} ; \mathrm{G}$ : rough fraction, $200-2000 \mu \mathrm{m} ; \mathrm{M}:$ medium fraction, $50-200 \mu \mathrm{m}$; $\mathrm{F}$ : fine fraction, $<50 \mu \mathrm{m}$, Gl : global fraction.

\begin{tabular}{|c|c|c|c|c|c|c|c|c|c|c|}
\hline & \multicolumn{10}{|c|}{ L'Etang des Noës } \\
\hline & \multicolumn{3}{|c|}{ Strate A } & \multicolumn{4}{|c|}{ Strate B } & \multicolumn{3}{|c|}{ Strate C } \\
\hline \multicolumn{2}{|c|}{ Fractions } & COT & NT & $\mathrm{C} / \mathrm{N}$ & COT & NT & $\mathrm{C} / \mathrm{N}$ & COT & NT & $\mathrm{C} / \mathrm{N}$ \\
\hline \multirow[t]{3}{*}{$\mathbf{G}^{\prime}$} & $a$ & $67,55 \pm 5,60$ & $3,39 \pm 0,31$ & 20.5 & $63,16 \pm 5,36$ & $3,05 \pm 0,15$ & 20,8 & $140,65 \pm 3,06$ & $4,25 \pm 0,75$ & 33 \\
\hline & b & $1.52 \pm 0.12$ & $0,07 \pm 0.01$ & & $4,01 \pm 0,34$ & $0,19 \pm 0,09$ & & $1,90 \pm 0,04$ & $0,06 \pm 0.01$ & \\
\hline & $\%$ & 6 & 5 & & 16 & 11,5 & & 11 & 4,5 & \\
\hline \multirow[t]{3}{*}{$\mathbf{G}$} & $\mathbf{a}$ & $68,30 \pm 7,47$ & $3,62 \pm 0,23$ & 18.9 & $46,88 \pm 5,27$ & $3,21 \pm 0,10$ & 14,6 & $52,45 \pm 3,84$ & $3.7 \pm 0.35$ & 14,2 \\
\hline & b & $8.74 \pm 0.92$ & $0,46 \pm 0,03$ & & $8,48 \pm 0,95$ & $0,58 \pm 0,02$ & & $1,51 \pm 0.11$ & $0.11 \pm 0.01$ & \\
\hline & $\%$ & 36 & 33 & & 35 & 35 & & 9 & 8,5 & \\
\hline \multirow[t]{2}{*}{$M$} & $\begin{array}{l}a \\
b\end{array}$ & $\begin{array}{c}29,93 \pm 2,48 \\
8,52 \pm 0,70\end{array}$ & $\begin{array}{l}1,70 \pm 0,22 \\
0,48 \pm 0,06\end{array}$ & 17,6 & $\begin{array}{c}30,20 \pm 2,69 \\
5,11 \pm 0,45\end{array}$ & $\begin{array}{l}1,92 \pm 0,13 \\
0,32 \pm 0,02\end{array}$ & 15,7 & $\begin{array}{c}47,06 \pm 4,11 \\
2,81 \pm 0,25\end{array}$ & $\begin{array}{r}3,05 \pm 0,25 \\
-0.19 \pm 0,01\end{array}$ & 15,4 \\
\hline & $\%$ & 35 & 34 & & 21 & 19 & & 17 & 15 & \\
\hline $\mathbf{F}$ & $\begin{array}{l}\mathbf{a} \\
\mathbf{b}\end{array}$ & $\begin{array}{l}9,85 \pm 0,86 \\
5,55 \pm 0,49\end{array}$ & $\begin{array}{l}0,70 \pm 0,05 \\
0,39 \pm 0,03\end{array}$ & 14 & $\begin{array}{c}11,70 \pm 0,96 \\
6,84 \pm 0,56\end{array}$ & $\begin{array}{l}0,99 \pm 0,04 \\
0,58 \pm 0,02\end{array}$ & 11,8 & $\begin{array}{l}11,74 \pm 0,99 \\
10,51 \pm 0,88\end{array}$ & $\begin{array}{l}1,05 \pm 0,05 \\
0,94 \pm 0,04\end{array}$ & 11,2 \\
\hline GI & $\begin{array}{l}\% \\
\text { b } \\
\%\end{array}$ & $\begin{array}{c}23 \\
24,33 \pm 2,23 \\
100\end{array}$ & $\begin{array}{c}28 \\
1.40 \pm 0.13 \\
100\end{array}$ & 17.2 & $\begin{array}{c}28 \\
24,44 \pm 2,30 \\
100\end{array}$ & $\begin{array}{c}34,5 \\
1,67 \pm 0.07 \\
100\end{array}$ & 14,6 & $\begin{array}{c}63 \\
16.74 \pm 1,28 \\
100\end{array}$ & $\begin{array}{c}72 \\
1,30 \pm 0,07 \\
100\end{array}$ & 12,9 \\
\hline
\end{tabular}


On notera cependant une décroissance de ce rapport des fractions grossières $G^{\prime}$ vers les fractions fines, au sein de chacune des strates, et la valeur particulièrement élevée de ce rapport dans la strate inférieure C de la fraction G'.

Le Réveillon (Tableau 4) : La teneur en carbone global est très importante dans la strate supérieure A' $(64,87 \mathrm{mg} / \mathrm{g})$ soit pratiquement trois fois supérieure à celle de la strate équivalente de l'étang des Noës. Cette teneur diminue pratiquement de moitié dans la strate inférieure $C^{\prime}(38,38 \mathrm{mg} / \mathrm{g})$. L'analyse de la teneur en carbone dans les différentes fractions granulométriques organo-minérales des deux strates montre que celle-ci est paradoxalement plus prépondérante dans la fraction G' de la strate inférieure $C^{\prime}(16,64 \mathrm{mg} / \mathrm{g}$ soit $43,5 \%)$ que dans la strate supérieure $A^{\prime}(14,15 \mathrm{mg} / \mathrm{g}$ soit $22 \%)$. Les fractions $G$ présentent une teneur similaire pour les deux strates $\left(19,12 \mathrm{mg} / \mathrm{g}\right.$ soit $29 \%$, strate $A^{\prime}$; $11,8 \mathrm{mg} / \mathrm{g}$ soit $31 \%$, strate $\left.C^{\prime}\right)$. Cependant, dans la strate supérieure $A^{\prime}$ la quantité de carbone piégée dans la fraction fine $F(19,97 \mathrm{mg} / \mathrm{g}$ soit $31 \%)$ est deux fois plus importante que celle de la strate C' $(6,27 \mathrm{mg} / \mathrm{g}$ soit $16 \%)$. Le taux d'azote de la strate supérieure $A^{\prime}$ est de $5,04 \mathrm{mg} / \mathrm{g}$ et diminue de $80 \%$ dans la strate inférieure C' $(1,72 \mathrm{mg} / \mathrm{g})$. Les répartitions en azote dans les différentes fractions granulométriques sont globalement équivalentes dans les deux strates. Le rapport $\mathrm{C} / \mathrm{N}$ est nettement supérieur dans les fractions de la strate inférieure
C' $(22,3)$ que dans celle de la strate supérieure $A^{\prime}$ $(12,9)$ et particulièrement dans les fractions $G^{\prime}$ (45 contre 20,2$)$ et $G(23,4$ contre 15,4$)$.

\subsubsection{Extraction alcaline des composés humiques}

La fraction grossière G' n'a pas été représentée sur les figures 5 et 6 du fait de sa faible importance dans toutes les fractions granulométriques et ce pour les deux milieux. Il en est de même pour la première fraction humique, les matières organiques légères (MOL), dans les tableaux 5 et 6. L'extraction des composés humiques se situe à $100 \% \pm 5 \%$, aussi les bilans ont été ajustés à $100 \%$ et répartis proportionnellement dans les différentes strates.

Etang des Noës : Les données exprimées en fonction de la texture indiquent (Fig. 5) que les strates $A$ et $C$ présentent une distribution opposée des teneurs en composés humiques en fonction des fractions granulométriques, la strate B présente quant à elle une distribution intermédiaire. En effet, dans la strate supérieure $\mathrm{A}$, les teneurs en composés humiques diminuent régulièrement de la fraction grossière $G$ vers la fraction fine $F$, alors que dans la strate inférieure $\mathrm{C}$, la teneur en composés humiques augmente très significativement de la fraction $G$ vers la fraction F. Pour la strate B, la teneur en composés humiques est maximale dans la fraction $G$, atteint une valeur minimale dans la fraction $M$, et atteint une valeur élevée dans la fraction $F$.

Tableau 4. Analyse de la matière organique dans les différentes fractions texturales organominérales des strates du sédiment de la rivière, Le Réveillon. ( $\mathrm{Cr}$. légende Tableau 3).

Table 4. Analysis of the organic matter in the various organo-mineral textural fractions of the sedimentary layers of the "Réveillon " (for legend see Table 3).

\begin{tabular}{|c|c|c|c|c|c|c|c|}
\hline & \multicolumn{7}{|c|}{ Le Réveillon } \\
\hline & \multicolumn{4}{|c|}{ Strate $A^{\prime}$} & \multicolumn{3}{|c|}{ Strate C' } \\
\hline \multicolumn{2}{|c|}{ Fractions } & $\mathrm{COT}$ & NT & $\mathrm{C} / \mathrm{N}$ & COT & NT & $\mathrm{C} / \mathrm{N}$ \\
\hline \multirow[t]{3}{*}{$\mathrm{G}^{\prime}$} & $a$ & $208,18 \pm 19,34$ & $10,29 \pm 0,96$ & 20,2 & $152,69 \pm 17,98$ & $3,42 \pm 0,26$ & 45 \\
\hline & b, & $14,15 \pm 1,31$ & $0,72 \pm 0,06$ & & $16,64 \pm 1,96$ & $0,38 \pm 6,03$ & \\
\hline & $\%$ & 22 & 14,5 & & 43 & 22 & \\
\hline \multirow[t]{3}{*}{ G } & $\mathbf{a}$ & $76,50 \pm 8,19$ & $4,98 \pm 0,41$ & 15,4 & $25,49 \pm 2,63$ & $1,09 \pm 0,10$ & 23,4 \\
\hline & b & $19,12 \pm 2,05$ & $1,24 \pm 0,10$ & & $11,80 \pm 1,21$ & $0,50 \pm 0,05$ & \\
\hline & $\%$ & 29 & 24,5 & & 31 & 29 & \\
\hline \multirow[t]{3}{*}{$\mathbf{M}$} & a & $40,66 \pm 3,08$ & $4,01 \pm 0,25$ & 10,1 & $19,94 \pm 1,91$ & $1,3 \pm 0,11$ & 15,34 \\
\hline & b & $11,63 \pm 0,88$ & $1,14 \pm 0,07$ & & $3,67 \pm 0,34$ & $0,24 \pm 0,0$ & \\
\hline & $\%$ & 18 & 22,5 & & 9,5 & 14 & \\
\hline \multirow[t]{3}{*}{$\mathbf{F}$} & a & $50,44 \pm 3,82$ & $4,92 \pm 0,21$ & 10,2 & $25,71 \pm 2,05$ & $2,34 \pm 0,17$ & 11 \\
\hline & b & $19,97 \pm 1,51$ & $1,94 \pm 0,08$ & & $6,27 \pm 0,50$ & $0,60 \pm 0,04$ & \\
\hline & $\%$ & 31 & 38,5 & & 16 & 35 & \\
\hline \multirow[t]{2}{*}{ Gl } & b & $64,87 \pm 5,75$ & $5,04 \pm 0,31$ & 12,9 & $38,38 \pm 4,01$ & $1,72 \pm 0,14$ & 22,3 \\
\hline & $\%$ & 100 & 100 & & 100 & 100 & \\
\hline
\end{tabular}


Tableau 5. Distribution des composés humiques dans les différentes fractions texturales organo-minérales des strates du sédiment de l'Etang des Noës. AF : acides fulviques; AH : acides humiques ; HUM : humine ; a : teneur (mg de carbone/g sec de la fraction granulométrique considérée), b : poids total dans chacune des fractions (a x \% texture organo-minérale de la fraction) et $\%$; pourcentage de chaque fraction ; G' : fraction très grossière $>2000 \mu \mathrm{m} ; \mathrm{G}:$ fraction grossière, $200-2000 \mu \mathrm{m} ; \mathrm{M}$ : fraction moyenne, $50-200 \mu \mathrm{m} ; \mathrm{F}:$ fraction fine, $<50 \mu \mathrm{m} ; \mathrm{Gl}:$ fraction globale.

Table 5. Distribution of humic compounds in the various organo-mineral textural fractions of the sedimentary layers of the « Etang des Noës » : AF: fulvic acids ; AH : humic acids ; HUM : Humin ; a : content (mg of carbon/dry gramme of the granulometric fraction considered), $b$ : total weight in each fraction (a $x \%$ organo-mineral texture) and $\%$; percentage of each fraction. $G^{\prime}$ : very rough fraction $>2000 \mu \mathrm{m} ; \mathrm{G}$ : rough fraction, $200-2000 \mu^{\mathrm{m}} ; \mathrm{M}:$ medium fraction, $50-200 \mu \mathrm{m} ; \mathrm{F}:$ fine fraction, $<50$ $\mu \mathrm{m} ; \mathrm{Gl}$ : global fraction.

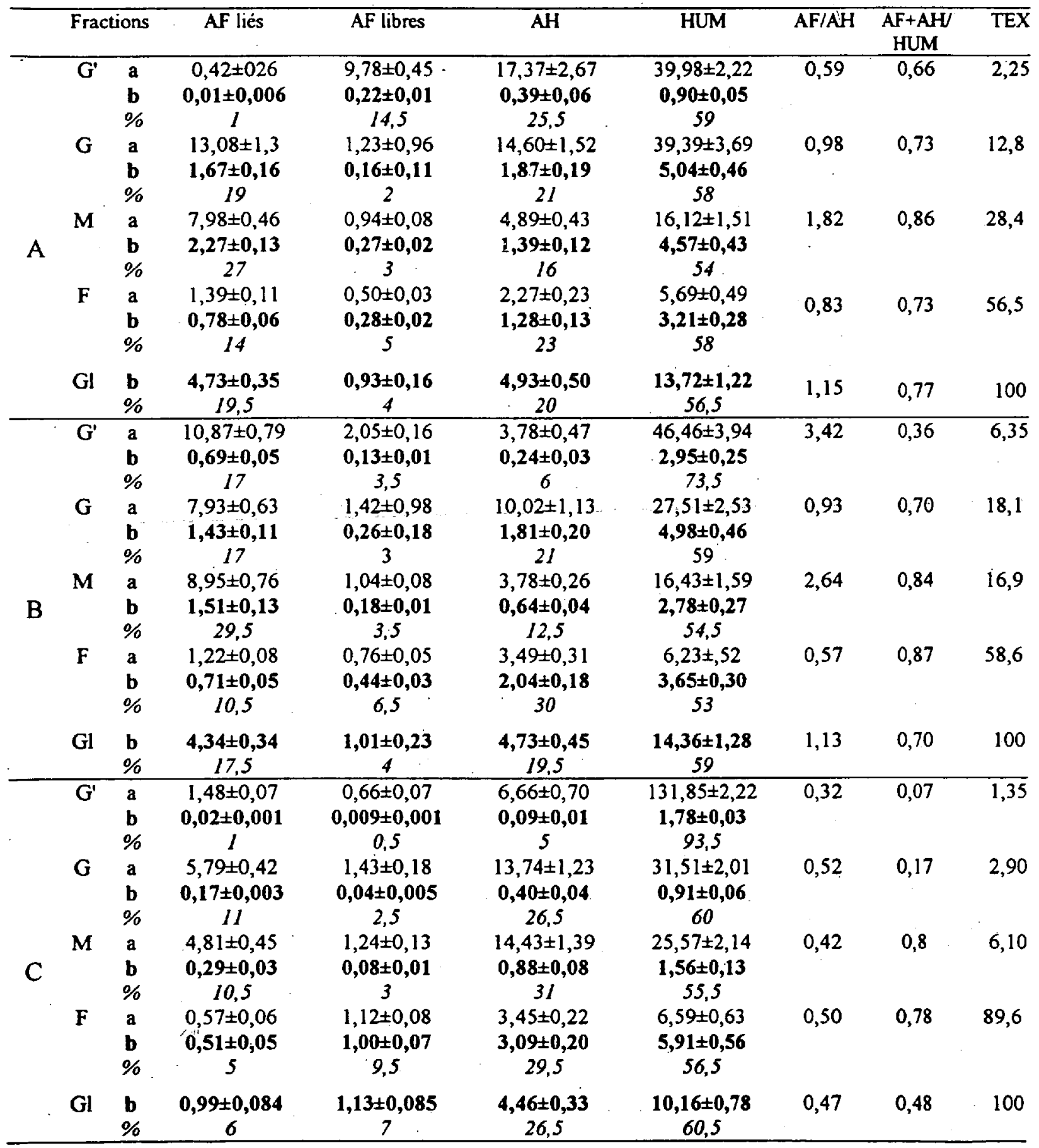


Tableau 6. Distribution des composés humiques dans les différentes fractions texturales organo-minérales des strates du sédiment de la rivière, Le Réveillon : (Cf légende Tableau 5).

Table 6. Distribution of humic compounds in the various organo-mineral textural fractions of the sedimentary layers of the « Réveillon " (for legend see Table 5).

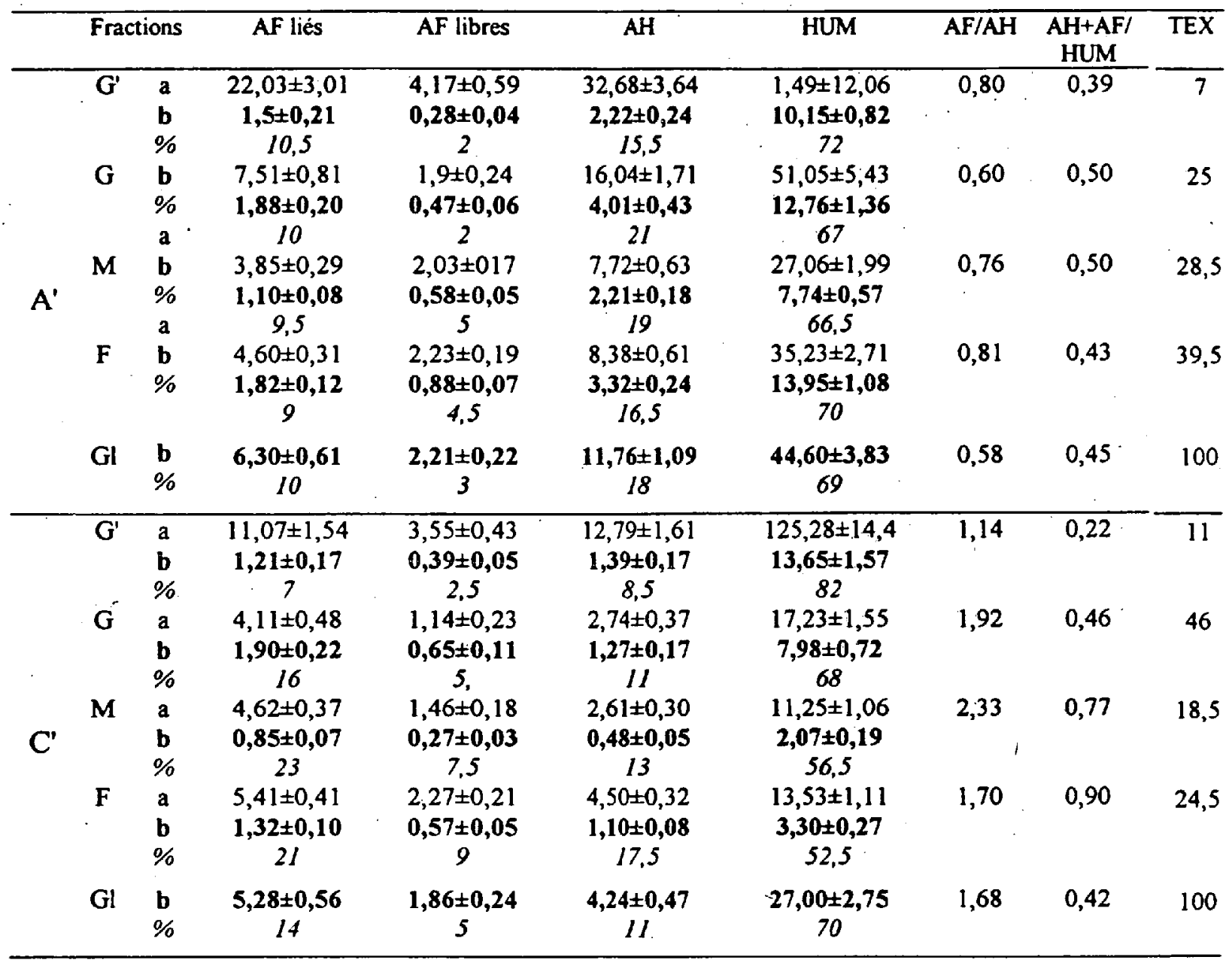

Les différences observées entre les différentes fractions granulométriques selon les strates sont dues au pourcentage relatif d'extraction des acides fulviques et humiques, les taux d'humine étant relativement comparables. Pour la strate supérieure $A$ et la strate intermédiaire $\mathrm{B}$, les rapports $\mathrm{AF} / \mathrm{AH}$ des composés humiques globaux n'enregistrent pas de variation significative $(1,15$ pour la strate $A$ et 1,13 pour la strate $B)$. En revanche, l'examen de ce rapport dans chaque fraction granulométrique montre pour ces deux strates que la fraction moyenne $M$ (200 à $50 \mu \mathrm{m}$ ) enregistre un plus fort taux d'extraction des acides fulviques que des acides humiques induisant respectivement des rapports $\mathrm{AF} / \mathrm{AH}$ de
1,82 pour la strate $A$ et de 2,64 pour la strate $B$. De plus, ce taux est également élevé dans la fraction $G$ ' de la strate intermédiaire $B$. En ce qui concerne la strate $\mathrm{C}$, le rapport $\mathrm{AF} / \mathrm{AH}$ des composés humiques globaux du sédiment est très faible $(0,47)$ et varie peu d'une fraction granulométrique à l'autre $(0,52$ pour $G ; 0,42$ pour $M$ et 0,50 pour $F$ ). Ces rapports très inférieurs à 1 sont révélateurs d'un plus fort pourcentage d'acides humiques que fulviques dans cette strate. Le rapport $(\mathrm{AF}+\mathrm{AH} / \mathrm{H})$ montre des valeurs similaires pour les trois strates $(0,77$ strate A ; 0,70 strate $B ; 0,66$ strate $C$ ), les valeurs les plus élevées étant constatées pour la fraction $\mathbf{M}$ des trois strates et $\mathrm{F}$ de la strate intermédiaire $\mathrm{B}$. 


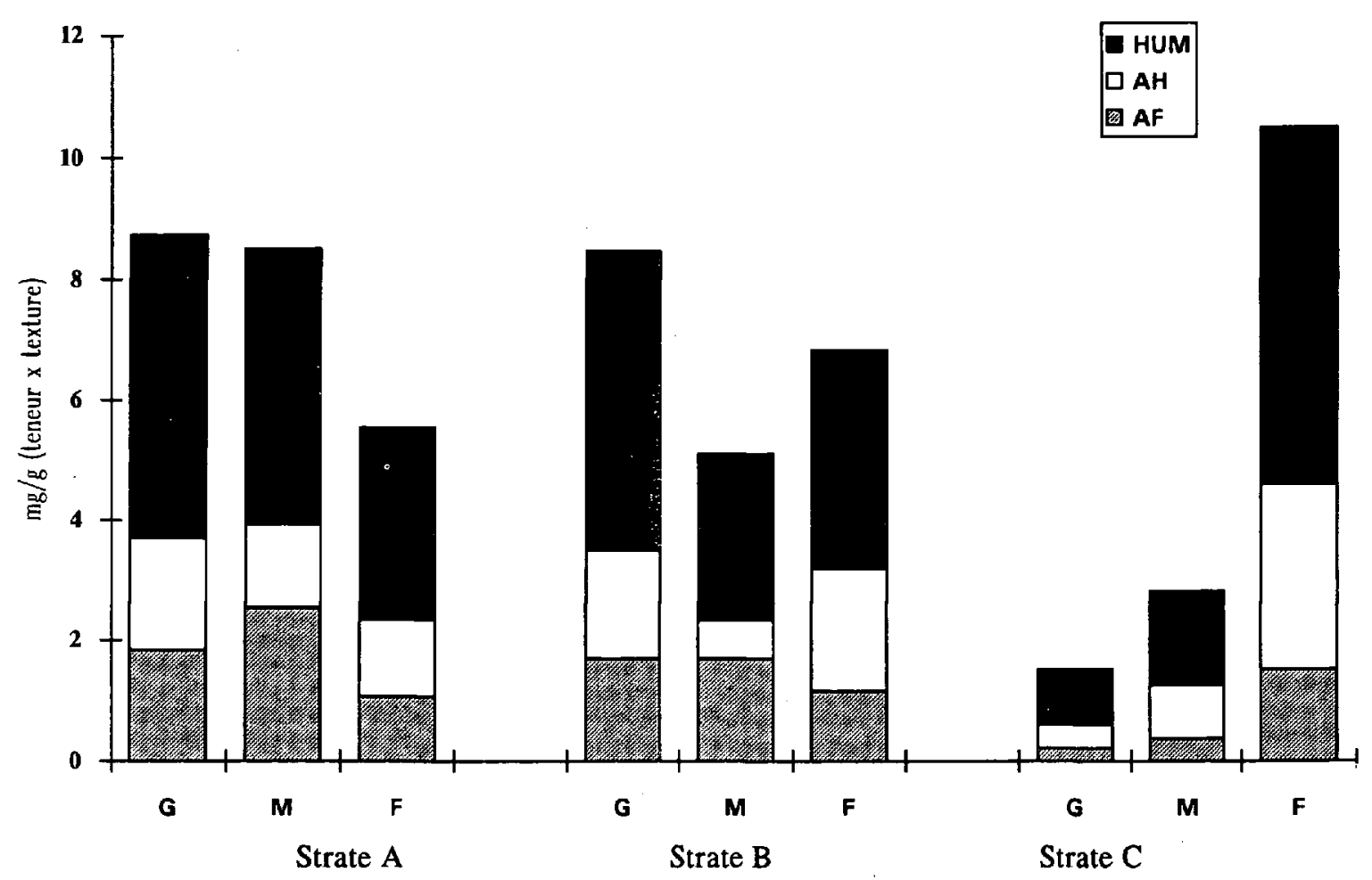

\section{L'Etang des Noës}

Fig. 5. Répartition des composés humiques dans les différentes fractions texturales organo-minérales des strates de l'étang des Noës (teneur $\mathrm{x}$ fraction texturale organo-minérale) $\mathrm{G}:$ fraction grossière, $200-2000 \mu \mathrm{m} ; \mathrm{M}:$ fraction moyenne, 50-200 $\mu \mathrm{m} ; \mathrm{F}:$ fraction fine, $<50 \mu \mathrm{m} ; \mathrm{HUM}$ : humine ; $\mathrm{AH}:$ Acides humiques ; AF : acides fulviques.

Fig. 5. Distribution of humic compounds in the various organo-mineral textural fractions of the layers of the Etang des Noës (content $\mathrm{x}$ organo-mineral textural fraction) $\mathrm{G}:$ coarse fraction, $200-2000 \mu \mathrm{m} ; \mathrm{M}:$ medium fraction, $50-200 \mu \mathrm{m} ; \mathrm{F}$ : fine fraction, $<50 \mu \mathrm{m}$; HUM : Humin ; AH : humic acids ; AF :'fulvic acids.

Le Réveillon : Les données exprimées en fonction de la texture indiquent (Fig. 6) que la strate A' présente une distribution en composés humiques prépondẻrante dans les fractions $G$ et $F$ et minimale dans la fraction $M$. La strate C' présente quant à elle une distribution maximale dans la strate $\mathrm{G}$ minimale dans la strate $M$ et intermédiaire dans la strate fine $F$.

Ces données permettent d'observer (Tableau 6) que le rapport $\mathrm{AF} / \mathrm{AH}$ des composés humiques globaux est inférieur à $1(0,58)$ dans la strate supérieure $A^{\prime}$, synonyme d'un fort pourcentage d'acides humiques, alors que celui-ci est supérieur à $1(1,68)$ dans la strate inférieure C' indiquant la présence d'un taux d'acides fulviques prépondérant. Il en est de même au sein de chacune des strates pour les différentes fractions granulométriques. La valeur du rapport $(\mathrm{AF}+$
$\mathrm{AH} / \mathrm{H}$ ) est sensiblement identique dans les quatre fractions $G^{\prime}, G, M$ et $F$ de la strate supérieure $A^{\prime}$, alors qu'elle diminue significativement de la fraction grossière $G^{\prime}$ à la fraction fine $F(0,22$ à 0,90$)$ dans la strate inférieure $C^{\prime}$. Le taux d'extraction des composés humiques est fonction de la taille des agrégats pour la strate inférieure.

\section{Discussion}

\subsection{Etang des Noës}

Cet étang est un plan d'eau que l'on peut qualifier aux vues des analyses physico-chimiques et biologiques de mésotrophe $\beta$ (Kolhwitz \& Marsen 1908). Ce milieu présente un transfert énergétique satisfaisant entre les différents maillons de la chaîne alimentaire. 


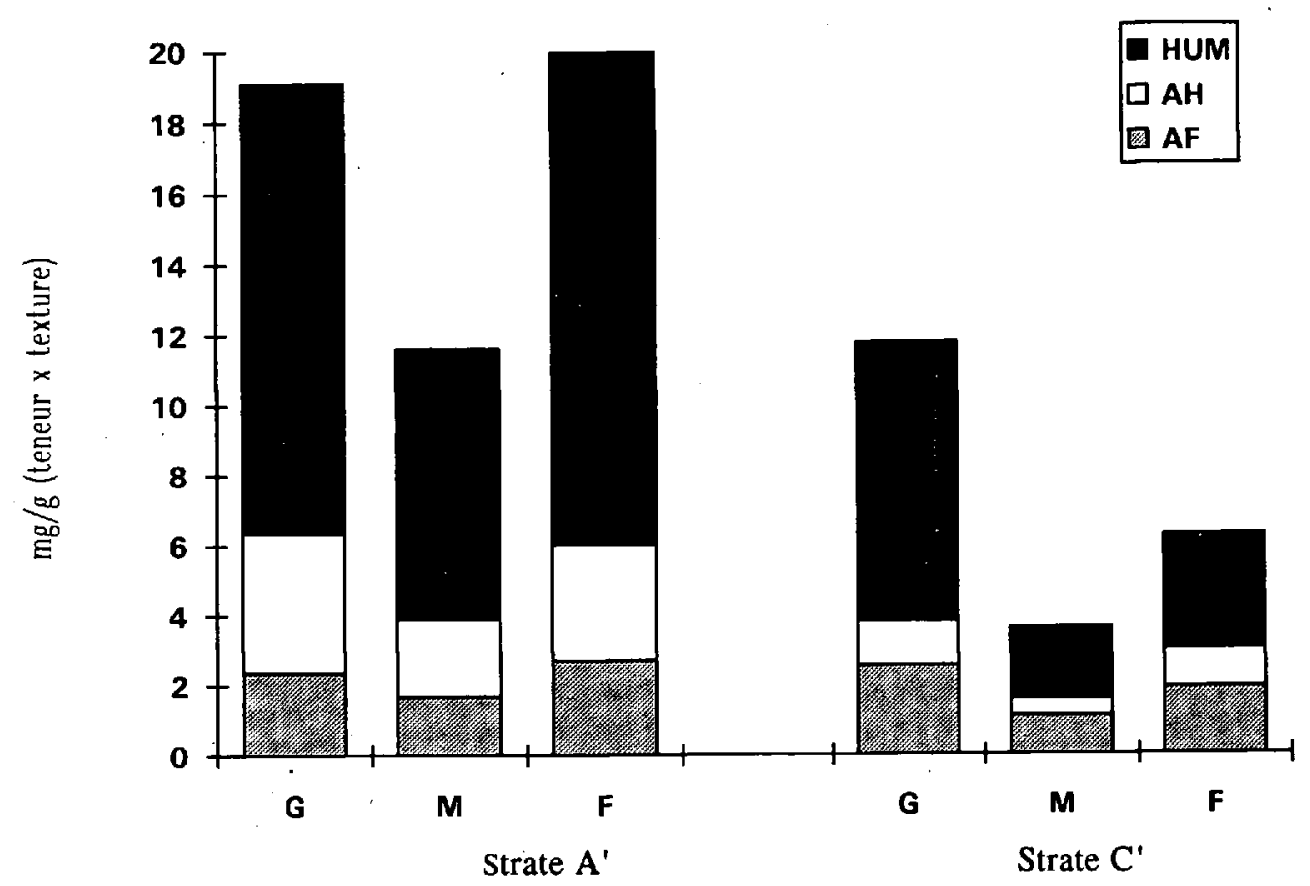

Le Réveillon

Fig. 6. Répartition des composés humiques dans les différentes fractions texturales organo-minérales des strates de la rivière, Le Réveillon (Cf légende Fig. 5).

Fig. 6. Distribution of humic compounds in the various organo-mineral textural fractions of the layers of the river « The Réveillon » (for legend see Fig. 5).

Cependant, le diagnostic hydrobiologique révèle une richesse faunistique spécifique faible tant à la fois sur le plan quantitatif que qualitatif. Ce phénomène n'est pas surprenant si l'on se réfère aux analyses physico-chimiques effectuées en mars 1992 plaçant globalement cet étang en classe de qualité 2 et en classe de qualité 3 , voire en hors classe, pour les paramètres tels que la DCO (Demande Chimique d'Oxygène) et la DBO5 (Demande Biologique d'Oxygène à 5 jours). La couverture végétale des berges semi-forestières à l'endroit des prélèvements est source d'apports en matières organiques non négligeables.

L'état d'oxydo-réduction de l'étang explicite son métabolisme et permet de prévoir les principales manifestations des échanges eau-sédiment. Les analyses montrent l'existence, dès les premiers centimètres du sédiment, d'une « microzone réduite » $($ Eh $<+200 \mathrm{mV})$ au sens de Bertru (1975). Celleci peut être due soit à une accélération de la consommation d'oxygène par les sédiments, soit à un renouvellement insuffisant de l'oxygène dans l'eau sus-jacente. L'étude de la respirométrie du sédiment montre que le dégagement de $\mathrm{CO} 2$ qui révèle des phénomènes de fermentation anaérobie est nettement plus important dans la strate profonde par rapport à la strate supérieure. L'existence d'une anaérobiose presque constante, sauf dans la couche épipélique où la présence de Thécamoebiens révèle des conditions aérobies, favorise la conservation des composés humiques. Selon Davaux (1976), le fait que la matière organique s'accumule en profondeur s'explique par une désoxygénation qui contribue à une meilleure conservation de cette matière à cause d'une diagénèse plus lente en milieu réducteur. Brinson et al. (1981) le confirment en précisant qu'un manque d'oxygène dissous limite la dégradation de la cellulose et la protège partiellement. Ce phénomène est d'ailleurs amplifié par la présence des composés humiques qui inhibent les processus de 
cellulolyse par complexation des produits intermédiaires de la dégradation de la cellulose (Blake 1985).

Le fractionnement granulométrique organominéral des sédiments suivi de l'analyse fine de la répartition de la matière organique par dosage des différents composés humiques permet de caractériser les compartiments où la matière organique subit des transformations. Rappelons, avant d'interpréter les résultats, que le terme "d'humine », à ce jour encore mal défini, correspond, dans la méthodologie utilisée dans cette étude, au carbone inextractible, sans préjuger de sa nature qui peut être d'origine variée. Plusieurs types d'humine peuvent être distinguées (Kononova 1966, Andreux 1978, Duchaufour 1983) : l'humine microbienne, formée de corps microbiens et de divers composés aliphatiques qui en dérivent, l'humine héritée, qui est une matière légère, proche de la matière végétale fraîche, formée de constituants des membranes, partiellement oxydés et liés aux argiles par des liaisons peu stables, l'humine d'insolubilisation, liée aux hydroxydes et à l'argile, et l'humine stabilisée résultant d'une évolution lente des acides humiques. Par simplification et dans la suite de la discussion nous distinguerons deux types d'humine pour formuler nos hypothèses, l'humine jeune qui regroupe l'humine microbienne et l'humine héritée et l'humine évoluée qui regroupe l'humine d'insolubilisation et l'humine stabilisée.

La strate supérieure A présente une localisation principale du carbone et de l'azote dans les fractions grossières G' et $G$ permettant de penser que la matière organique peu dégradée de taille $>200 \mu \mathrm{m}$ est piégée dans ces fractions. L'analyse du carbone des composés humiques extraits des fractions $G$ et G' montre une part importante d'humine. Cette humide est vraisemblablement composée, en majeure partie, d'humine jeune. La fraction fine $F$ indique que le processus d'humification est déjà amorcé conduisant à une production d'acides fulviques et humiques. Ceux-ci sont produits en quantité équivalente (AF/AH global proche de 1).

La strate profonde $\mathrm{C}$ présente des quantités de carbone et d'azote inférieures à celles de la strate A mettant en évidence les phénomènes de minéralisation qui tendent à diminuer le stock de matière organique en fonction du temps. La quantité de carbone correspondant à des particules organiques frấches de taille $>200{ }_{\mu} \mathrm{m}$ n'est pas totalement négli- geable. Il s'agit là de matière organique bloquée non fractionnée de façon mécanique, du fait de l'absence de macroinvertébrés pour lesquels les conditions d'anaérobiose existant dans cette strate sont très défavorables. Cette strate ne permet que l'activité microbienne anaérobie. Le carbone est dans cette strate très nettement localisé dans la fraction argileuse F. Cet état indique un fractionnement important de la matière organique située en profondeur. La quantité d'acides humiques plus conséquente que celle des fulviques $(\mathrm{AF} / \mathrm{AG}<1)$ ainsi que la présence d'une grande quantité d'humine dans cette fraction fine confirment que le processus d'humification est fortement engagé dans cette strate conduisant à la formation de molécules formées par polymérisation croissante des composés humiques (Andreux 1978, Stevenson \& Goh 1971). De plus, ces composés humiques doivent être ici fortement stabilisés du fait de la texture minérale à forte teneur en argile de ce milieu. La formation de complexes organo-minéraux entre les molécules humiques et les argiles constitue certainement un obstacle à la minéralisation de ces molécules.

La strate B présente globalement les caractéristiques évolutives intermédiaires entre les strates $\mathbf{A}$ et C. La quantité de matière organique contenue dans la fraction grossière $\mathrm{G}$ est encore très importante et révèle une grande quantité d'humine jeune, humine qui provient sans aucun doute des fragments végétaux, encore reconnaissables à l'œil nu, qui se localisaient en majorité dans cette strate. Dans la fraction fine, le taux important de composés humiques révèle que le processus d'humification est largement initié.

Si l'on pose comme hypothèse qu'aucune perturbation anthropique n'a affecté le sédiment depuis 18 ans, la comparaison entre chaque strate mettrait en évidence une évolution graduelle de la surface du sédiment vers la profondeur qui se traduit par une polymérisation croissante des macromolécules humiques due à une évolution des processus d'humification. Les teneurs en composés humiques montrent que la fraction fine $F$ de la strate supérieure $A$ est quantitativement la moins riche par rapport aux fractions plus grossières. En revanche, la strate inférieure $C$ enregistre un fort taux de carbone dans sa fraction fine. Cette répartition des teneurs indique que la strate inférieure $C$ est plus évoluée que la strate supérieure A. La voie de l'humification préférentielle dans la strate $C$ révèle un ralentissement 
dans le fonctionnement de l'écosystème s'opposant à la remise en circulation immédiate des éléments nutritifs dans le milieu et donc au «turn over » de la matière organique. La texture minérale limonoargileuse dans les trois strates de ce milieu est à mettre directement en relation avec la quantité de composés humiques piégés et stabilisés dans les fractions fines des différentes strates.

\subsection{Le Réveillon}

Les résultats des analyses physico-chimiques et biologiques révèlent une mauvaise qualité des eaux du Réveillon sur le secteur où ont été fait les prélèvements (DCO, DB05, 02 dissous). Ceci est essentiellement dû à la forte quantité de matières organiques en suspension dans l'eau. Le fort envasement constaté à cet endroit provient de la végétation rivulaire et des rejets de la station d'épuration, sources importantes de matières organiques, et qui, du fait de l'absence de courant à cet endroit, sédimentent à proximité de leur relargage.

Les études de respirométrie ont montré l'état très réduit des sédiments profonds. Les teneurs en carbone et en azote évoluent différemment selon la taille des particules et la profondeur.

La strate supérieure $A^{\prime}$ montre un taux très important de carbone. Ce taux est majoritairement distribué dans le compartiment humine, en particulier dans les fractions G' et $G$ mais également dans la fraction $F$. L'humine des fractions $G$ ' et $G$ est vraisemblablement une humine jeune, reflétant ainsi une grande quantité de matières organiques peu dégradées (Qualls \& Haines 1990). En revanche, celle de la fraction fine $F$ est probablement constituée, en partie, d'humine plus évoluée. La répartition des composés humiques dans les quatre fractions granulométriques montre que dans cette couche superficielle, le processus dominant n'est pas une minéralisation mais déjà une humification conduisant à la formation de molécules complexes et stables. On peut donc supposer qu'une partie de l'humine est ici piégée sous forme d'agrégats organo-minéraux. Les rapports $\mathrm{AF} / \mathrm{AH}$ restent inférieurs à 1 dans les quatre fractions G', G, M, F. Le profil évolutif de cette strate serait à rapprocher de celui de la strate intermédiaire de l'étang des Noës, avec cependant, un processus d'humification plus accentué.

Les taux de carbone et d'azote dans la strate $C^{\prime}$ sont deux fois moins importants que dans la strate
A'. Il est délicat dans ce cas précis de formuler des hypothèses. En effet, le dépôt des sédiments de la strate $\mathrm{C}$ est-il antérieur ou postérieur à l'installation de la station d'épuration? De plus, les rejets de la station sont-ils chargés de façon homogène en matières organiques au cours du temps ? Si la strate $C^{\prime}$ est le reflet de l'évolution de la strate $A$ ' il faut admettre qu'un fort processus de minéralisation se déroule au sein de la vase, ce qui parait incompatible avec les processus biochimiques décrits pour les sols hydromorphes. En raison de la forte anaérobiose qui ralentit considérablement l'activité biologique, la décompositon de la matière organique fraîche de ces sols est très lente et incomplète, et l'on constate que les apports annuels excédent les pertes par minéralisation (Menut 1974). On peut donc, dans le cas des sédiments du Réveillon, proposer comme hypothèse, du fait de l'état anaérobique constaté: dans: le sédiment inférieur, que le dépôt de la strate: C' est antérieur à l'installation de la station d'épuration. Manifestement, la dégradation de la matière organique paraît bloquée dans cette strate conme l'atteste les faibles taux d'AH et d'AF et la forte valeur du rapport $C / N$. Les rapports $A F / A H$ sont supérieurs à 1 dans les quatre fractions granulométriques, indiquant une faible polycondensation des molécules humiques.

\section{Conclusion}

Cette étude a montré que le long d'un profil sédimentaire, l'analyse du carbone et la caractérisation des différents composés humiques en fonction des fractions granulométriques organo-minérales permettent de rendre compte de l'état de la matière organique dans ces compartiments. L'état d'oxydation du sédiment joue un rôle prépondérant dans le déroulement de la dégradation de la matière organique par l'activité des macroinvertébrés et de la microflore. Dans ces conditions d'anaérobiose, comme c'est le cas pour les sédiments du Réveillon et dans une moindre mesure de l'étang des Noës, seule l'activité microbienne peut intervenir dans les processus de dégradation. D'autre part, la texture minérale du sédiment joue un rôle important dans le processus de stabilisation des composés humiques formés, notamment dans l'élaboration des complexes argilo-humiques. La comparaison des deux milieux montre que, dans le cas de l'étang des Noës, où un curage a eu lieu en 1974 , des processus d'humification se déroulent le long du profil 
sédimentaire, la matière organique de la strate inférieure présentant des composés humiques fortement polymérisés. En revanche, dans le cas des sédiments du Réveillon, milieu anaérobie dès les premiers centimètres, la voie de l'humification est prépondérante dès la strate superficielle. Dans la strate profonde, la dégradation de la matière organique paraît bloquée. Ce blocage se traduit par une faible production des acides fulviques et humiques. Le faible taux de matières organiques dans cette strate ainsi que sa granulométrie argileuse pourraient indiquer que le dépôt de la strate inférieure est antérieur à la mise en place de la station d'épuration. Ces analyses mettent en exergue que l'état physico-chimique d'un horizon profond n'est pas le seul résultat des conditions environnementales dans lesquelles il se trouve actuellement, particulièrement en milieu anthropisé, et que sa diagénèse doit être parfaitement maîtrisée pour comprendre son évolution.

\section{Travaux cites}

Andreux F. 1978: - Genèse et propriétés des molécules humiques. In Pédologie, Tome 2 : Constituants et propriétés du sol, Bonneau M. \& Souchier B. (eds) : 97-122.

Andreux F. 198:1. - Utilisation de molécules de synthèse dans l'étude des: processus d'insolubilisation et de biodégradation des polycondensats humiques. Bull. A.F.E.S. Science du Sol, 4 : $271-29.0$.

Anne P. 1945. - Détermination du carbone total. Ann. Agro., $15: 1611-172$.

Bertru G. I975. - Etudes physico-chimique et microbiologique des échanges sédiment-eau : influence sur la trophie du système et la periodicité du phytoplancton. Thèse de doctorar, Université de Rennes: 239 p.

Blake G. 1985. - Les macrophytes lacustres : production et dêcomposition. Thèse de doctorat, Université de Grenoble : $138 \mathrm{p}$.

Brinson M.M., Lugo A.E. \& Brown S. 1981. - Primary productivity, decomposition and consumer activity in freshwater wetlands. Ann. Rev. Ecol. Syst., $12: 123-16$ I.

Bruckert S., Hetier J.M. \& Guitierrez F. 1974. - Dynamique de l'humification des andosols du Massif Central français et des Canaries. Caractérisation physico-chimique des complexes. organo-minéraux. Bulletin Association Française Etude Sols; $4: 225-245$.

Buffle J.A.E. 1977, - Les substances humiques et leurs interactions avec les ions minéraux. T.S.M. L'eou, $1: 3-1.1$.

Choudry C. 1983. - Sorptive interactions with environmental chemical toxics. Environ. Chem., $6: 127-171$.

Christman R.F. \& Gjessing E.T. 1983. - Aquatic and terrestrial humic materials. Michigan : Ann Arbor Science Publishers : $538 \mathrm{p}$.

Dabin B. 1976. - Méthode d'extraction et fractionnemeñ des matières humiques des sols. Application à quelques études pédologiques et agronomiques dans les sols tropicaux. Cah. O.R.S.T.O.M., sér. Pédol., 4 : 287-297.
Davaux E. 1976. - Contribution à l'étude géochimique et sédimentologique des dépôts lacustres récents. Thèse de doctorat, Université de Genève : $129 \mathrm{p}$.

Duchaufour P. 1970. - Précis de pédologie. 3ème édition, Masson, Paris, 481 p.

Duchaufour P. 1983. - Pédologie I : Pédogénèse et classification. Sous la direction de Duchaufour P. \& Souchier B., 2ème édition, Masson, Paris : $491 \mathrm{p}$.

Duchaufour P. \& Jacquin F. 1966. - Nouvelles recherches sur l'extraction et le fractionnement des composés humiques. Bull. ENSA Nancy, $8: 3-24$.

Faust S.D. \& Hunter J.V. 1971. - Organic compounds in aquatic environments. Marcel Dekker INC, New York : 145 p.

Feller C. 1979. - Une méthode de fractionnement granulométrique de la matière organique des sols : application aux sols tropicaux à textures grossières, très pauvres en humus. Cah. O.R.S.T.O.M. sér. Pédol., 17 : 339-346.

Feller C., Bernhardt-Reversat F., Garcia J.L., Pantier J.J., Roussos S. et Van Vliet-Lanoe B. 1983. - Etude de la matière organique de différentes fractions granulométriques d'un sol sableux tropical. Effet d'un amendement organique (compost). Cah. O.R.S.T.O.M. sér. Pédol., 3 : 223-238.

Flaig W. 1970. - Contribution à la connaissance de la constitution et de la synthèse des acides humiques. Science du Sol. $2: 39-72$.

Fustec C., Chauvet E. \& Gas G. 1989. - Lignin degradation and humus formation in alluvial soils and sediments. Applied and Environmental Microbiology : 922-926.

Gjessing E.T. 1976. - Physical and chemical characteristics of aquatic humus. Ann Arbor Science Publishers, Michigan : $238 \mathrm{p}$.

Guy R.F., Chakrabarti C.L. \& Schramm L.L. 1975. - The application of a simple chemical model of natural waters to metal! fixation in particulate matter. Can. J. Chem., 53 : $661-667$.

Haworth R.D. 1971. - The chemical nature of humic acid. Soil Scii, 111: 71-79.

Ishiwatari I. 1985. - Geochemistry of humic substances in lake sediments. In Humic substances in soil, sediment and water, Aiken G.R., D.M. Mcknight, R.L. Wershaw and P. Maccanthy, eds, Wiley-interscience publication, New York : $147-180 \mathrm{p}$.

Jamagne M. 1967. - Bases et techniques d'une cartographie des sois. Ann. Agron., N ${ }^{\circ}$ hors série, Vol $18: 142$ p.

Kolltuitz K. \& Marsen M. 1908. - Okologie des tierischen soprobien. Internat. Rev. Hydrobiol., 2 : 126-152.

Kononova M.M. 1966. - Soil Onganic matter. 2nd Edition. Pergamon Press, London, 544 p.

Menut G. 1974. - Recherches écologiques sur l'évolution de la matière organique des sols tourbeux. Thèse Doctorat, Université de Nancy : $124 \mathrm{p}$.

Malcom R.L. 1990. - The uniqueness of humic substances in each of soil, stream and marine environments. Analytica Chimica Acta, 232 : 19-30.

Munsell Soil Colors Charts. 1990. - Macbeth (ed.), New York.

Ong H.J. \& Busque R.E: 1968. - Coagwlation of humic colloids by metals ions. Soil Sci., 106: 220-224.

Povoledo D. \& Goltermann H.L. 1975s. - Humic substances. Center of agricultural publishing: and Documentation, Wageningen : $312 \mathrm{p}$. 
Qualls R.G. \& Haines B.L. 1990. - The influence of humic substances on the aerobic decomposition of submerged leaf litter. Hydrobiologia, 206 : 133-138.

Schnitzer M. \& Kahn S.U. 1978. - Soil organic matter. Elsevier, Amsterdam : 327 p.

Senesi N. \& Testini C. 1982. - Physico-chemical investigations of interaction mechanisms between S-Triazine herbicides and humic acids. Geoderma, 28 : 129-146.
Stevenson F.J. 1982. - Humus chemistry : genesis, composition and reaction. Wiley Interscience, New York : $330 \mathrm{p}$.

Stevenson F.J. \& Goh (K.M.). 1971. - Infrared spectra of humic acids and related substances. Geochim., Cosmochim., Acta, $35: 417-483$.

Tan K.H. 1974. - The catalytic decomposition of clay minerals by complex reaction with humic and fulvic acids. Soil Sci., $120: 188-194$. 